Pacific Journal of Mathematics

AFFINE CURVES OVER AN ALGEBRAICALLY NONCLOSED
FIELD 


\title{
AFFINE CURVES OVER AN ALGEBRAICALLY NON-CLOSED FIELD
}

\author{
Maria Grazia Marinari, Francesco OdetTi \\ AND MARIO RAIMONDO
}

In this paper, different $\bar{k}$-completions of a curve over an algebraically non-closed field $k$ are compared. If the curve has $k$-points at infinity, then it is shown to admit a completion which is canonical. If $k=\mathbf{R}$ this is true also for rational curves.

Introduction. If $\left(V, \Theta_{V}\right)$ is an affine algebraic curve defined over a field $k(k \neq \bar{k})$ then $V$ is the set of $k$-points of several non-isomorphic $\bar{k}$-curves which are called completions of $V$.

In this paper we compare these different completions and we prove (Theorem 3.1.) the existence of completions which, without creating new singularities, are extended as large as possible in the sense that they are not affine open sets of larger ones. These completions are called minimal. If a curve satisfies the condition of having $k$-points "at infinity" (cf. Theorem 3.5) then these minimal completions turn out to be all isomorphic. We shall say that "this is the canonical" completion of the curve. In the case $k=\mathbf{R}$ we prove that the above condition is also necessary if the genus of the curve is bigger than zero, while rational affine real curves always admit canonical complexification.

The involved techniques are mainly those of A. Tognoli (cf. [6]). We emphasize the use of affine representations (rather than that of completions (cf. §0)). First we introduce a suitable partial ordering in the set of isomorphism classes of affine representations; we are then able to show that the subset of affine representations which correspond to completions having no non-rational singularities is sufficiently rich and has minimal (up to isomorphism) elements.

0. We first recall some preliminaries. Throughout this paper $k$ denotes a field and $\bar{k}$ an algebraic closure of it. Let $V$ be an algebraic subset of $k^{n}$ and let $\theta_{V}$ denote the sheaf of regular functions defined over the open sets of $V$. It is known that

$$
\Gamma_{V}=\Gamma\left(V, \vartheta_{V}\right)=N_{V}^{-1}\left(k\left[X_{1}, \ldots, X_{n}\right] / \mathcal{T}_{V}\right)
$$

where $\mathcal{T}_{V}=\left\{P \in k\left[X_{1}, \ldots, X_{n}\right] \mid P_{\mid V} \equiv 0\right\} \quad$ and $\quad N_{V}=\{g \in$ $k\left[X_{1}, \ldots, X_{n}\right] / \mathcal{T}_{V} \mid g(x) \neq 0$ for each $\left.x \in V\right\}$. More generally an affine 
$k$-variety $V=\left(V, \theta_{V}\right)$ is a topological space $V$ plus a sheaf $\theta_{V}$ of $k$-valued functions on $V$ which is isomorphic to an irreducible algebraic subset of some $k^{n}$ plus its sheaf of regular functions (see [6] for general references).

A criterion for a $k$-algebra to be the ring of global regular functions of some affine variety defined over $k$ is given in [5]. Ibidem it is also showed that the global sections functor gives an equivalence of categories between the category of affine $k$-varieties and the opposite to the full subcategory of " $k$-algebras" consisting of those objects that satisfy the quoted above criterion.

Given now an affine algebraic $k$-variety $V$, each embedding $i: V \rightarrow k^{n}$ gives rise to $j: V \rightarrow \bar{k}^{n}$ ([6], Def. 1. p. 28) and we call completion of $V$ (complexification if $k=\mathbf{R}$ ) the algebraic variety $\tilde{V}$ which is the closure of $j(V)$ into $\bar{k}^{n}$. Note that different embeddings of $V$ in $k^{n}$ may induce different completions of $V$. We shall study a completion $\tilde{V}$ of $V$ mainly considering the ring $P_{V}$ of regular functions from $\tilde{V}$ to $k$. Observe that $P_{V} \otimes_{k} \bar{k}$ is the ring of coordinates of $\tilde{V}$ and that $\Gamma_{V} \simeq N^{-1} P_{V}$ (where $N=\left\{g \in P_{V} \mid g(x) \neq 0\right.$ for all $\left.x \in V\right)$. The ring $P_{V}$ is said to be an affine representation of $\Gamma_{V}$. The affine representations have been characterized as follows ([5] Prop. 2.1):

Proposition 0.1. Let $V$ be an affine k-variety. $A$-algebra $A$ is an affine representation of $\Gamma_{V}$ if and only if

(i) $A$ is of finite type over $k$,

(ii) $\cap_{\mathfrak{N} \in \operatorname{Specm}_{k^{A}} A} \mathfrak{N}=(0)$ and $\operatorname{Specm}_{k} A \simeq V$,

(iii) $\Gamma_{V} \stackrel{\simeq}{\simeq} N_{A}^{-1} A$ where $N_{A}=\{a \in A \mid a \notin \Re$ for each $\Re \in$ $\left.\operatorname{Specm}_{k} A\right\}$.

REMARK 0.2. If $A$ is an affine representation of some $\Gamma_{V}$, then:

(a) for every $\Re \in \operatorname{Specm}_{k} A$ we have $A_{\Re} \simeq\left(\Gamma_{V}\right)_{\Re^{e}}$

(b) if $\operatorname{dim}_{k} V=1$, then for every $\mathfrak{p} \in \operatorname{Specm} A-\operatorname{Specm}_{k} A$ we have $(A-\mathfrak{p})^{-1} \Gamma_{V} \simeq \operatorname{Fract}\left(\Gamma_{V}\right)$.

We point out that if $V=\left(V, \mathcal{O}_{V}\right)$ is an affine irreducible curve defined over $k$, then $K=\operatorname{Fract}\left(\Gamma_{V}\right)(\simeq$ Fract $A$ for each affine representation $A$ ) is a field of algebraic functions in one variable over $k$.

From now on, unless contrarily specified, we shall always consider affine irreducible curves and $\mathfrak{V}$ will denote the set of all valuations of $K$ over $k$.

The terms valuation, divisor, genus of $K$ will be used with the same meaning as in [1]. For every $v \in \mathcal{T},\left(R_{v}, \Re_{v}\right)$ will indicate the corresponding local ring with its maximal ideal and $\operatorname{deg} v$ will indicate the integer $\left[R_{v} / \Re_{v}: k\right]$. 
For each affine representation $A$ of $\Gamma_{V}$, we introduce the following non-negative integers:

$$
\begin{aligned}
& \Xi(A)=\#\left\{v \in \mathcal{V} \mid R_{v} \not \supset A\right\}, \\
& \Xi_{n}(A)=\#\left\{v \in \mathcal{V} \mid R_{v} \not \supset A \text { and } \operatorname{deg} v=n\right\} .
\end{aligned}
$$

Remarks 0.3. (a) $0<\Xi(A)<\infty$ (cf. [4] Lemma 6.5 Ch. I) and so $\Xi_{n}(A)=0$ for large $n$.

(b) there exists a one-to-one correspondence which to each maximal ideal $\mathfrak{N} \in \operatorname{Specm}_{k} A-\operatorname{Sing}(\operatorname{Spec} A)$ associates a valuation ring $R_{v}$ such that deg $v=1, R_{v} \supset A$ and $R_{v} \simeq A_{\text {丹 }}$. So, as $\operatorname{Specm}_{k} A \simeq \operatorname{Specm}_{k} \Gamma_{V}=$ Specm $\Gamma_{V}, \Xi_{1}(A)$ depends only on $V$ and we shall denote this integer by $\Xi_{1}(V)$.

1. We remark that there exist affine representations of non-singular $k$-varieties such that the corresponding completions have singularities as $\bar{k}$-varieties:

EXAMPLE 1.1. The R-algebra

$$
A=\mathbf{R}\left[\left(X^{2}+1\right)\left(X^{2}+4\right), X\left(X^{2}+1\right)\left(X^{2}+4\right), X^{3}+7 X\right]
$$

is an affine representation of the ring of regular algebraic functions on the real line $\mathbf{A}_{\mathbf{R}}^{1}$ and $A \otimes_{\mathbf{R}} \mathbf{C}$ is the ring of coordinates of a complex curve having two ordinary nodes.

This induces us to introduce among all the affine representations of a fixed $\Gamma_{V}$ the following distinction.

Definition 1.2. An affine representation $A$ of $\Gamma_{V}$ is said to be a good affine representation if $\operatorname{Sing}(\operatorname{Spec} A) \subset \operatorname{Specm}_{k} A$.

It is possible to associate to each affine representation $A$ of $\Gamma_{V}$ a good one such that its corresponding completion is a "partial normalization" of the completion associated to $A$.

THEOREM 1.3. Let $A$ be an affine representation of $\Gamma_{V}$ and let $A^{\prime}$ be its integral closure in $\Gamma_{V}$. Then $A^{\prime}$ is a good affine representation of $\Gamma_{V}$.

Proof. First of all, by using criterion (0.1) we show that $A^{\prime}$ is an affine representation of $\Gamma_{V}$. Clearly $A^{\prime} \subset \bar{A}$, where $\bar{A}$ is the integral closure of $A$ in $K$, then $A^{\prime}$ is a $k$-algebra and an $A$-module of finite type as $\bar{A}$ is so and 
$A$ is noetherian. The inclusions $A \hookrightarrow A^{\prime} \hookrightarrow \Gamma_{V}$ induce $V=\operatorname{Specm} \Gamma_{V}=$ $\operatorname{Specm}_{k} \Gamma_{V} \rightarrow \operatorname{Specm}_{k} A^{\prime} \rightarrow \operatorname{Specm}_{k} A$ whose composite is a homeomorphism and $\operatorname{Specm}_{k} A^{\prime}$ maps injectively into $\operatorname{Specm}_{k} A$ (use [5], Th. 2.2.). On the other hand it is easy to check that $\Gamma_{V}=N_{A^{\prime}}^{-1} A^{\prime}$. If now $a=$ $\cap \Re^{\prime}\left(\Re^{\prime} \in \operatorname{Specm}_{k} A^{\prime}\right)$, then $\mathfrak{a} \Gamma_{V}=\left(0_{\Gamma_{V}}\right)$, so $\mathfrak{a}=\left(0_{A^{\prime}}\right)$, since $\Gamma_{V}$ is a ring of fractions of $A^{\prime}$. All this shows our claim. To prove that $\operatorname{Sing}\left(\operatorname{Spec} A^{\prime}\right) \subset \operatorname{Specm}_{k} A^{\prime}$, let $\mathfrak{p}^{\prime} \in \operatorname{Specm} A^{\prime}-\operatorname{Specm}_{k} A^{\prime}$ and let $\mathfrak{p}=$ $\mathfrak{p}^{\prime} \cap A \in \operatorname{Specm} A-\operatorname{Specm}_{k} A$. Then $(A-\mathfrak{p})^{-1} A^{\prime}$ is integrally closed in $(A-\mathfrak{p})^{-1} \Gamma_{V} \simeq K$. Since $\left(A^{\prime}-\mathfrak{p}^{\prime}\right)^{-1} A^{\prime}$ is a ring of fractions of $(A-\mathfrak{p})^{-1} A^{\prime}$, it follows that $\mathfrak{p}^{\prime} \notin \operatorname{Sing}\left(\operatorname{Spec} A^{\prime}\right)$.

Proposition 1.4. An affine representation $A$ of $\Gamma_{V}$ is good if and only if $A$ is integrally closed in $\Gamma_{V}$.

Proof. After (1.3) we only have to show that if $\operatorname{Sing}(\operatorname{Spec} A) \subset$ Specm $_{k} A$ then $A=A^{\prime}$, the integral closure of $A$ in $\Gamma_{V}$. Let $\mathfrak{f}^{\prime}=$ $\operatorname{Ann}_{A}\left(A^{\prime} / A\right)$ be the conductor of $A^{\prime}$ in $A$ (resp. let $\mathfrak{f}=\operatorname{Ann}_{A}(\bar{A} / A)$ be the conductor of $\bar{A}$ in $A$ ). Clearly $A=A^{\prime}$ iff $\mathfrak{f}^{\prime}=\left(1_{A}\right)$, therefore we need to prove that every $\mathfrak{p} \in \operatorname{Specm} A$ does not contain $\mathfrak{f}^{\prime}$. If $\mathfrak{p} \in \operatorname{Specm}_{k} A$ then $A_{\mathfrak{p}} \simeq(A-\mathfrak{p})^{-1} \Gamma_{V} \simeq(A-\mathfrak{p})^{-1} A^{\prime}$ and so $\mathfrak{p} \not \supset \mathfrak{f}^{\prime}$. On the other hand if $\mathfrak{p} \in \operatorname{Specm} A-\operatorname{Specm}_{k} A$ then $\mathfrak{p} \not \supset \mathfrak{f}$ by hypothesis, therefore $\mathfrak{p} \not \supset \mathfrak{f}^{\prime}$ as $\mathfrak{f} \subset \mathfrak{f}^{\prime}$

REMARK 1.5. If $V$ is smooth and $A$ is a good affine representation of $\Gamma_{V}$, then both $A$ and $\Gamma_{V}$ are Dedekind domains and it is easy to check that:

(a) $\Gamma_{V}=\cap R_{v}$ (intersection running throughout the set of $R_{v}$ 's such that $R_{v} \supset \Gamma_{V}$ and $\left.\operatorname{deg} v=1\right)$.

(b) $A \cong \Gamma_{V} \cap\left(\cap R_{v}\right)$ (the second intersection now running through the set of $R_{v}$ 's such that $R_{v} \supset A$ and $\operatorname{deg} v>1$ (cf. Rem. $0.3 \mathrm{~b}$ ).

2. We introduce now a partial ordering $\prec$ on the set of (isomorphism classes of) affine representations of a given $\Gamma_{V}$.

Definition 2.1. If $A$ and $B$ are affine representations of a given $\Gamma_{V}$ we say that $A$ precedes $B(A \prec B)$ if there exists an isomorphism $\alpha: N_{A}^{-1} A \rightarrow$ $N_{B}^{-1} B$ such that its restriction $\left.\alpha\right|_{A}$ maps $A$ into $B$. Plainly $\prec$ is reflexive and transitive, so it remains to show that it is antisymmetric, i.e. if $A \prec B$ and $B \prec A$ then $A \simeq B$. We shall prove this first for good affine representations, then for all ones.

LEMMA 2.2. If $A$ and $B$ are affine representations of a given $\Gamma_{V}$ such that $A$ is good and $A \prec B$ via $\alpha: A \rightarrow B$, then $\alpha$ is a flat ring homomorphism. 
Proof. The induced embedding $\bar{\alpha}: \bar{A} \rightarrow \bar{B}$ is flat since $\bar{A}$ is Dedekind and $\bar{B}$ is an overring of $\bar{A}$. It is enough to show that for every $\mathfrak{p} \in \operatorname{Spec} B$, $B_{\mathfrak{p}}$ is flat over $A_{\mathrm{q}}$ where $\mathfrak{q}=\mathfrak{p} \cap A$. For this:

(i) if $\mathfrak{p} \in \operatorname{Sing}(\operatorname{Spec} B)$ then $\mathfrak{p} \in \operatorname{Specm}_{k} B$ since $B$ is good, therefore $A_{\mathfrak{q}} \simeq B_{\mathfrak{p}} \simeq\left(\Gamma_{V}\right)_{\mathfrak{p}^{e}}$.

(ii) if $\mathfrak{p} \notin \operatorname{Sing}(\operatorname{Spec} B)$ then $q \notin \operatorname{Sing}(\operatorname{Spec} A)$ since $\alpha^{*}$ : $\operatorname{Sing}(\operatorname{Spec} B)$ $\rightarrow \operatorname{Sing}(\operatorname{Spec} A)$ is a homeomorphism. Hence $B_{\mathfrak{p}} \simeq(B-\mathfrak{p})^{-1} \bar{B} \simeq$ $\bar{B}_{\mathrm{p}}$ and $A_{\mathrm{q}} \simeq(A-\mathfrak{q})^{-1} \bar{A} \simeq \bar{A}_{\mathrm{q}}$ e. Our contention follows from the fact that $\bar{B}_{\mathfrak{p}^{e}}$ is flat over $\bar{A}_{\mathfrak{q}^{e}}$ and $\mathfrak{q}^{e} \overline{\mathrm{q}} \simeq \mathfrak{p}^{e}$.

Remarks 2.3. (a) The above result fails if $A$ is not good. For instance let $A$ be the affine representation of the real line given in example (1.1) and let $B=\mathbf{R}[X]$. It is clear that $A \prec B$ but $B$ is not flat over $A$, which in fact is not good.

(b) The above lemma is also false if $\operatorname{dim}_{k} V>1$, even if the involved completions are smooth. For example let $A=\mathbf{R}[X, Y]$ (affine representation of $\mathbf{A}_{\mathbf{R}}^{2}$ ) and let $\phi: A \rightarrow A$ be the $\mathbf{R}$-homomorphism given by $\phi(X)=X$, $\phi(Y)=\left(X^{2}+1\right) Y$. Plainly $\phi$ extends to an isomorphism $\tilde{\phi}: \Gamma_{\mathbf{A}_{\mathbf{R}}^{2}} \rightarrow \Gamma_{\mathbf{A}_{\mathbf{R}}^{2}}$, but it is not flat.

LEMMA 2.4. Let $A$ and $B$ be affine representations of a given $\Gamma_{V}$. Then

(i) if $A \prec B$ then $\Xi(A) \leq \Xi(B)$.

Let in addition $A$ and $B$ be both good. Then

(ii) if $A \prec B$ and $\Xi(A)=\Xi(B)$ then $A \simeq B$,

(iii) if $A \prec B$ and $B \prec A$ then $A \simeq B$.

Proof. (i) Let $\phi: A \rightarrow B$ be the inclusion given by $A \prec B$. Then $\phi$ extends to an automorphism $\tilde{\phi}: \Gamma_{V} \rightarrow \Gamma_{V}$ and therefore to an automorphism $\Phi: K=\operatorname{Fract}(A) \rightarrow \operatorname{Fract}(B)=K$ such that $\Phi(A) \subset B$. Since $\Xi(A)=\Xi(\Phi(A))$ and $\left\{v \in \mathscr{V} \mid R_{v} \not \supset \Phi(A)\right\} \subset\left\{v \in \mathscr{V} \mid R_{v} \not \supset B\right\}$; we get the wanted inequality.

(ii) Let now $\phi^{*}$ be the induced map on the spectra. Clearly we need only to prove that $\phi^{*}$ is a homeomorphism. Now $\phi^{*}$ maps $\operatorname{Sing}(\operatorname{Spec} B)=$ $\left\{\Re_{1}, \ldots, \Re_{r}\right\} \subset \operatorname{Specm}_{k} B$ bijectively into $\operatorname{Sing}(\operatorname{Spec} A)=\left\{\mathfrak{\Re}_{1}, \ldots, \Re_{r}\right\}$ $\subset \operatorname{Specm}_{k} A$. Therefore, for every $i, i=1, \ldots, r$, we have $A_{\Re_{1}} \simeq A_{\phi^{*}\left(\Re_{j}\right)} \simeq$ $B_{\Re_{j}}$ for some $j, j=1, \ldots, r$. On the other hand, for every $\Re \in \operatorname{Spec} A-$ $\operatorname{Sing}(\operatorname{Spec} A), A_{\Re} \simeq R_{v}$ for some $v \in \mathcal{V}$ and $R_{v} \simeq \Phi\left(R_{v}\right)=R_{w}$ for some $w \in \mathcal{V}$ (by (i), since $\Xi(A)=\Xi(B)$ ). Now $R_{w} \simeq B_{\Re}$ for some $\Re \in$ Specm $B-\operatorname{Sing}(\operatorname{Spec} B)$ and $\Re=\phi^{*}(\Re)$. So $\phi^{*}$ turns out to be surjective; it is also an open immersion since it is flat (by 2.2.) and birational (by hypothesis), thus $\phi^{*}$ is a homeomorphism.

(iii) It follows immediately from (i) and (ii). 
THEOREM 2.5. The relation $\prec$ is a partial ordering on the set of all affine representations of a given $\Gamma_{V}$.

Proof. We need only to check that $\prec$ is antisymmetric. Let $A \prec B$ via $\phi: A \rightarrow B$ and $B \prec A$ via $\psi: B \rightarrow A$. Consider the following commutative diagram

$$
\begin{array}{ccccc}
\Gamma_{V} \simeq N_{A}^{-1} A & \stackrel{\sim}{\rightarrow} & N_{B}^{-1} B & \stackrel{\sim}{\rightarrow} & N_{A}^{-1} A \simeq \Gamma_{V} \\
\uparrow & & \uparrow & & \uparrow \\
A & \stackrel{\phi}{\rightarrow} & B & \stackrel{\psi}{\rightarrow} & A
\end{array}
$$

We want to show that $\eta=\psi \cdot \phi$ is an isomorphism. Call $\tilde{\eta}$ the isomorphism on the top row (which turns out to be induced by $\eta$ ). It is easy to check that its restriction $\eta^{\prime}=\left.\tilde{\eta}\right|_{A^{\prime}}$ to the integral closure of $A$ in $\Gamma_{V}$ maps $A$ into itself. Therefore by (2.4) $\eta^{\prime}$ is an isomorphism. The map $\eta^{*}: \operatorname{Spec} A$ $\rightarrow$ Spec $A$ induced by $\eta$ is a homeomorphism on an open set since it is birational, on the other hand $\eta^{*}: \operatorname{Spec} A^{\prime} \rightarrow \operatorname{Spec} A^{\prime}$ is injective so $\eta^{*}$ is also surjective. Our thesis follows then like in Lemma 2.4 (ii).

3. We show now that in order to find a sort of canonical completion the right context seems to be given by the set of good affine representations.

THEOREM 3.1. The partial ordering $\prec$ has minimal elements on the set of good affine representations of a given $\Gamma_{V}$.

Proof. If $A \prec B$ strictly, by (2.4) $\Xi(A)<\Xi(B)$. Therefore, because of the finiteness of $\Xi$, there exist minimal affine representations corresponding to the minimal values of $\Xi$.

We point out that $\prec$ is an ordering which is not inductive in the ascending way even if we consider only good affine representations:

EXAMPLE 3.2. Consider the R-algebras $A_{n}=\mathbf{R}\left[X, \Pi_{j=1}^{n} 1 /\left(X^{2}+j^{2}\right)\right]$ for $n>0$. The $A_{n}$ 's give rise to an ascending chain of good affine representations of $\Gamma_{\mathbf{A}_{\mathbf{R}}^{\prime}}$ which is clearly non-stationary. We also remark that $\prec$ is not inductive in the descending way on the set of all affine representations:

EXAMPLE 3.3. We generalize example (1.1) by constructing a strictly descending chain $\cdots \prec A_{n} \prec A_{n-1} \prec \cdots \prec A_{1} \prec A_{0}=\mathbf{R}[t]$ of affine representations of $\mathbf{A}_{\mathbf{R}}^{1}$ where $A_{n}$ is obtained inductively from $A_{n-1}$ by glueing 
pairwise the complex points $(2 n-1) i$ with $2 n i$ and $-(2 n-i) i$ with $-2 n i$. We get this as an explicit application of the theory of glueings developed in [3], namely $A_{n}$ is the pullback in the diagram:

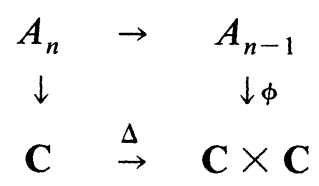

where $\Delta$ is the diagonal map and, for every $f \in A_{n-1}, \phi(f)=$ ( $f(2 n i), f(2 n-1) i)$. Straightforward but tedious computations give the following explicit form for the $A_{n}$ 's: let $h \in\{1, \ldots, n\}$ and let

$$
\begin{aligned}
F_{h}(t) & =\left(t^{2}+(2 h-1)^{2}\right)\left(t^{2}+(2 h)^{2}\right), \\
G_{h, n}(t) & =(2 h(2 h-1)-n) t^{3}+h(2 h-1)(4 h(2 h-1)+1+2 n) t, \\
X_{n}(t) & =\prod_{h=1}^{n} F_{h}(t), \quad Y_{n}(t)=t X_{n}(t), \\
T_{h, n}(t) & =\left(\prod_{j=1}^{h-1} F_{j}(t)^{n-h+1}\right) \cdot\left(\prod_{s=h+1}^{n} F_{s}(t)^{n-s+1}\right) \cdot\left(\prod_{r=h}^{n} G_{h, r}\right) .
\end{aligned}
$$

Then $A_{n}=\mathbf{R}\left[X_{n}, Y_{n}, T_{1, n}, \ldots, T_{n, n}\right]$. Thus the rings $A_{n}$ are affine representations of the ring of regular functions on $\mathbf{A}_{\mathbf{R}}^{\mathrm{l}}$ which have the following properties: (i) $A_{n}^{\prime}=\mathbf{R}[t]$; (ii) the complexification of $\mathbf{A}_{\mathbf{R}}^{1}$ corresponding to $A_{n}$ has exactly $2 n$ singular non-real points; (iii) $A_{n+1} \prec A_{n}$ and $\cdots \prec A_{n}$ $\prec \cdots \prec A_{1} \prec A_{0}\left(A_{0}=\mathbf{R}[t]\right)$ is a non-stationary descending chain.

Definition 3.4. An affine representation $A$ of $\Gamma_{V}$ is said to be canonical if it is good and $A \prec B$ for every good affine representation $B$.

Clearly the canonical representation is defined up to isomorphism. The corresponding completion will be called canonical completion; we shall see $(\$ 4)$ that it does not always exist. Here we give a sufficient criterion. First we remark that if $\Gamma_{V}$ has canonical representation $A$, then $\Xi(A)$ is minimal in the set $\{\Xi(B) \mid B$ good affine representation $\}$. Furthermore, since $\Xi_{1}(B)=\Xi_{1}(V)$ is independent of the representation $B, A$ is minimal with respect to $\prec$ if $\sum_{i=1}^{\infty} \Xi_{i}(A)$ is minimal.

THEOREM 3.5. Let $V$ be a smooth curve such that $\Xi_{1}(V) \neq 0$. Then $\Gamma_{V}$ admits a canonical representation. 
Proof. Let $A$ be a good representation which is minimal with respect to $\prec$. We claim that $\Xi_{l}(A)=0$ for all $i>1$. Suppose not. Let $\mathcal{V}$ be the abstract Riemann surface of $K$ over $k$. Fix an embedding $\sigma: A \rightarrow \Gamma_{V} \subset K$, then there exists a valuation $\bar{v} \in \mathcal{V}$ with $\operatorname{deg} \bar{v}=i>1$ such that $R_{\bar{v}} \not \supset$ $\sigma(A)$. By assumption there are some valuations $v$ such that $\operatorname{deg} v=1$ and $\sigma(A) \not \subset R_{v}$ (since $\left.\Gamma_{V} \not \subset R_{v}\right)$ and the divisor associated with $\{v \in \mathscr{V} \mid \operatorname{deg} v$ $\left.=1, \sigma(A) \not \subset R_{v}\right\}$ is ample so that a suitable multiple of it gives an embedding of $V$ in $k^{m}$. Let $C$ be the affine representation determined by this embedding. It is easy to check that $C \simeq \cap R_{v}\left(R_{v} \supset C\right)$, but $C \subset R_{\bar{v}}$ so that $C \prec A$, contradiction with the minimality of $A$. This proves the claim.

Suppose now that $B$ is another minimal representation of $\Gamma_{V}$; by the claim we have $\Xi_{l}(B)=0$ for each $i>1$. Fix $\tau: B \rightarrow \Gamma_{V} \subset K$ then $\{v \in$ $\left.\mathfrak{V} \mid R_{v} \supset \tau(B)\right\}=\left\{v \in \mathcal{V} \mid R_{v} \supset \sigma(A)\right\}$, therefore

$$
A \simeq \sigma(A)=\bigcap R_{v}\left(R_{v} \supset \tau(B)\right)=\tau(B) \simeq B .
$$

CoRollary 3.6. Let $V$ be as in Theorem 3.5 and let $A$ be a good affine representation. Then $A$ is the canonical representation if and only if $\Xi_{i}(A)=0$ for all $i>1$.

4. We conclude this note applying the above results to the case $k=\mathbf{R}$.

If $V$ is a smooth real curve, then $\mathbf{R}$ is the field of constants of $K=$ Fract $\Gamma_{V}$ over $\mathbf{R}$ itself and the valuations of $K$ over $\mathbf{R}$ have only degrees 1 or 2 . We call genus of $V(g(V))$ the genus of $K$. Since we always take for granted that $V$ has $\mathbf{R}$-points then $V$ has genus 0 iff it is rational (cf. [1] Ch. II§2).

REMARK 4.1. Let $V$ be a smooth real curve. In the following cases $V$ has canonical complexification.

(i) $\Xi_{1}(V) \neq 0$. In fact from Theorem 3.5 it turns out that the canonical complexification $\tilde{V}$ of $V$ is $\operatorname{Spec}\left(P_{V} \otimes_{\mathrm{R}} \mathrm{C}\right)$ where $P_{V}=\cap R_{v}$ (all $v \in \mathcal{V}$ but those of degree 1 such that $R_{v} \not \supset \Gamma_{V}$ ).

(ii) $\Xi_{1}(V)=0$ and $V$ is rational. In fact if $A$ is a minimal good affine representation of $\Gamma_{V}$, then $A$ is of the form $S^{-1}\left(\mathbf{R}[X, Y] /\left(Y^{2}+C(X)\right)\right)$ where $S$ is a multiplicative set, $C(X)$ is a polynomial of degree 2 with two distinct factors in $\mathbf{C}[X]$ and $Y^{2}+C(X)$ is irreducible (see e.g. [2] §3). By the minimality of $A$, we may assume $S=U\left(\mathbf{R}[X, Y] /\left(Y^{2}+C(X)\right)\right)$ (otherwise $\Xi_{1}(A) \neq 0$ ). Actually $C(X)$ factors in $\mathbf{R}[X]$ (or else $\operatorname{Specm}_{\mathbf{R}} A$ would be empty). Thus, after a change of coordinates, we can assume 
$A=\mathbf{R}[X, Y] /\left(X^{2}+Y^{2}-1\right)$. Therefore all good minimal affine representations of $\Gamma_{V}$ are isomorphic.

The cases of Remark 4.1 are the only possible ones as follows from the following.

Proposition 4.2. Let $V$ be a smooth real curve such that $\Xi_{1}(V)=0$ and $g(V) \geq 1$. Then there exist infinitely many non-isomorphic complexifications corresponding to the minimal good affine representations of $V$.

Proof. Consider first the case $g(V)=1$. Let $A$ be an affine representation of $\Gamma_{V}$ such that $\Xi(A)=\Xi_{2}(A)$ and let $\bar{V}$ be a non-singular projective closure of the complexification of $V$ associated to $A$. Let $\sigma$ be the conjugation in $\bar{V}$ and let $\{P, \sigma P\}$ be the support of the divisor at infinity of $\operatorname{Spec}\left(A \otimes_{\mathbf{R}} \mathbf{C}\right)$ in $\bar{V}$ which consists of two complex conjugated points. Fixed a group law + in $\bar{V}$, any automorphism of $\bar{V}$ is a translation followed by a group automorphism. Namely if $\phi \in \operatorname{Aut}(\bar{V}), \phi(x)=\varepsilon x+$ $x^{\prime}$ where $\varepsilon$ is a (fourth or sixth) root of 1. Clearly there exist (infinitely many) points $Q$ 's such that $\sigma Q-Q \neq \varepsilon(\sigma P-P)$ for all $\varepsilon$, for which $\phi(\sigma P) \neq \sigma Q$ whenever $\phi(P)=Q$. Choose one of these $Q$ 's and let $w$ be the valuation of degree two associated to $Q+\sigma Q$. Then the ring $B=$ $\bigcap_{v \neq w} R_{v}$ is a minimal representation and $B \neq A$ otherwise an isomorphism between $A$ and $B$ would extend to an automorphism of $\bar{V}$ sending $\{Q, \sigma Q\}$ into $\{P, \sigma P\}$.

If $g(V)>1$, the group of automorphisms of any projective closure of a complexification of $V$ has order at most $84(g-1)$. The same argument as above will prove our contention.

All together we have proved:

THEOREM 4.3. A smooth affine real curve $V$ has canonical complexification if and only if it is either rational or embeddable as a non-compact algebraic subspace of some $\mathbf{R}^{n}$ (in the usual topology).

\section{REFERENCES}

1. C. Chevalley, Introduction to the theory of algebraic functions in one variable, Math. Surveys, 6 Amer. Math. Soc., (1951).

2. W. Cunnea, Unique factorization in algebraic function fields, Illinois J. Math., 8 (1964), 425-438.

3. P. Gianni, Morfismi interi delle varietà algebriche, Boll. U.M.I. (5) 15-A (1978); $187-196$.

4. R. Hartshorne, Algebraic Geometry, Springer (1977). 
5. M. G. Marinari-M. Raimondo, Properties of the regular functions ring of affine varieties defined over any field, Rend. Sem. Mat. Univ. e Polit. Torino, 37, 1 (1979), 63-70.

6. A. Tognoli, Algebraic Geometry and Nash Functions, Instit. Math. vol. III Academic Press (1978).

Received October 18, 1980.

ISTITUTO DI MATEMATICA

Via L. B. Alberti 4

I-16132 GENOVA, ITALY 


\section{PACIFIC JOURNAL OF MATHEMATICS}

\section{EDITORS}

Donald BabbitT (Managing Editor)

University of California

Los Angeles, CA 90024

Hugo Rossi

University of Utah

Salt Lake City, UT 84112

C. C. Moore and Arthur Ogus

University of California

Berkeley, CA 94720
J. DugunduI

Department of Mathematics

University of Southern California

Los Angeles, CA 90089-1113

R. FinN and H. SAmelson

Stanford University

Stanford, CA 94305

\section{ASSOCIATE EDITORS}
R. ARENS
E. F. BECKENBACH
B. H. NEUMANN
F. WOLF
K. YosHIDA (1906-1982)

\section{SUPPORTING INSTITUTIONS}

UNIVERSITY OF ARIZONA

UNIVERSITY OF BRITISH COLUMBIA

CALIFORNIA INSTITUTE OF TECHNOLOGY

UNIVERSITY OF CALIFORNIA

MONTANA STATE UNIVERSITY

UNIVERSITY OF NEVADA, RENO

NEW MEXICO STATE UNIVERSITY

OREGON STATE UNIVERSITY
UNIVERSITY OF OREGON

UNIVERSITY OF SOUTHERN CALIFORNIA

STANFORD UNIVERSITY

UNIVERSITY OF HAWAII

UNIVERSITY OF TOKYO

UNIVERSITY OF UTAH

WASHINGTON STATE UNIVERSITY

UNIVERSITY OF WASHINGTON 


\section{Pacific Journal of Mathematics}

Vol. 107, No. $1 \quad$ January, 1983

John Kelly Beem and Phillip E. Parker, Klein-Gordon solvability and the

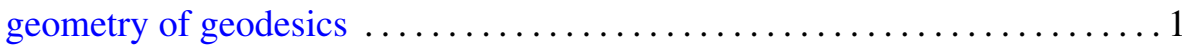

David Borwein and Amnon Jakimovski, Transformations of certain

sequences of random variables by generalized Hausdorff matrices ..... 15

Willy Brandal and Erol Barbut, Localizations of torsion theories . . . . . . . 227

John David Brillhart, Paul Erdős and Richard Patrick Morton, On sums

of Rudin-Shapiro coefficients. II ........................... 39

Martin Lloyd Brown, A note on tamely ramified extensions of rings $\ldots \ldots \ldots 71$

Chang P'ao Ch'ên, A generalization of the Gleason-Kahane-Żelazko

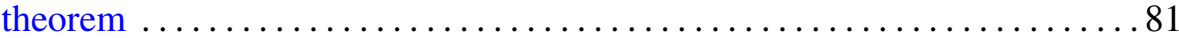

I. P. de Guzman, Annihilator alternative algebras $\ldots \ldots \ldots \ldots \ldots \ldots$. . . . 89

Ralph Jay De Laubenfels, Extensions of $d / d x$ that generate uniformly

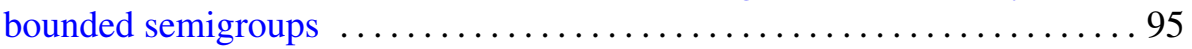

Patrick Ronald Halpin, Some Poincaré series related to identities of $2 \times 2$

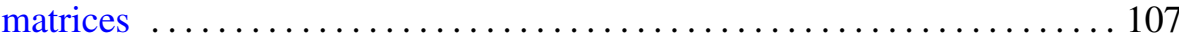

Fumio Hiai, Masanori Ohya and Makoto Tsukada, Sufficiency and

relative entropy in $*$-algebras with applications in quantum systems . . . 117

Dean Robert Hickerson, Splittings of finite groups $\ldots \ldots \ldots \ldots \ldots \ldots \ldots 14$

Jon Lee Johnson, Integral closure and generalized transforms in graded

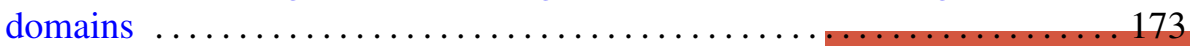

Maria Grazia Marinari, Francesco Odetti and Mario Raimondo, Affine

curves over an algebraically nonclosed field . ................. 179

Douglas Shelby Meadows, Explicit PL self-knottings and the structure of

PL homotopy complex projective spaces $\ldots \ldots \ldots \ldots \ldots \ldots \ldots \ldots \ldots$

Charles Kimbrough Megibben, III, Crawley's problem on the unique

$\omega$-elongation of $p$-groups is undecidable .................... 205

Mary Elizabeth Schaps, Versal determinantal deformations $\ldots \ldots \ldots \ldots 213$

Stephen Scheinberg, Gauthier's localization theorem on meromorphic

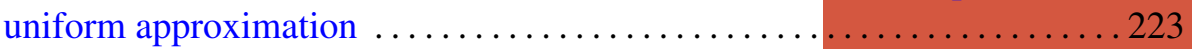

Peter Frederick Stiller, On the uniformization of certain curves . . . . . . . 229

Ernest Lester Stitzinger, Engel's theorem for a class of algebras . . . . . . . . 245

Emery Thomas, On the zeta function for function fields over $F_{p} \ldots \ldots \ldots 251$ 